\title{
Density distributions and form factors of the exotic ${ }^{8} \mathrm{~B}$ nucleus
}

\author{
R. A. Radhi, A. K. Hamoudi, W. Z. Majeed \\ Department of Physics, College of Science, University of Baghdad, Baghdad- Iraq \\ E-mail: wasan_zmz@yahoo.com
}

\begin{abstract}
Results of charge, neutron and matter densities and related form factors for one- proton halo nucleus ${ }^{8} \mathrm{~B}$ are presented using a twofrequency shell model approach. We choose a model space for the core of ${ }^{7} \mathrm{Be}$ different from that of the extra one valence proton. One configuration is assumed for the outer proton to be in $1 p_{1 / 2}$ - shell. The results of the matter density distributions are compared with those fitted to the experimental data. The calculated proton and matter density distributions of this exotic nucleus exhibit a long tail behavior, which is considered as a distinctive feature of halo nuclei. Elastic electron scattering form factors of this exotic nucleus are also studied. The effects of the proton halo on the electron scattering form factors are analyzed. The form factors of the exotic unstable ${ }^{8} \mathrm{~B}$ nucleus are compared with those of the stable ${ }^{10} \mathrm{~B}$ nucleus. The differences between the results of unstable ${ }^{8} \mathrm{~B}$ and stable ${ }^{10} \mathrm{~B}$ nuclei come from the long tail in the density distribution of the last proton.
\end{abstract} Key words

Proton-rich exotic nuclei, Shell model calculations, Nucleon density distributions of halo nuclei.

\author{
Article info \\ Received: Sep. 2012 \\ Accepted: Jan. 2013 \\ Published: Apr. 2013
}

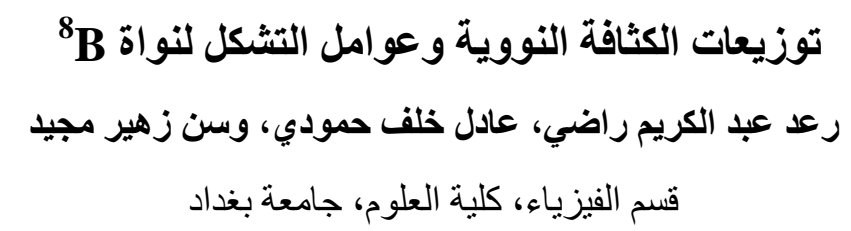

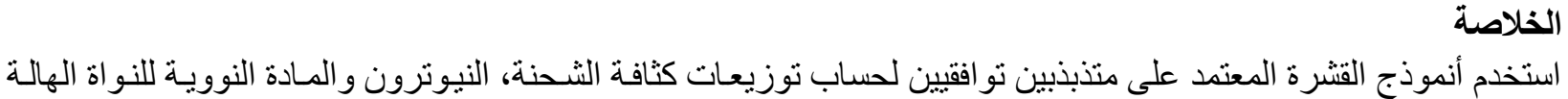

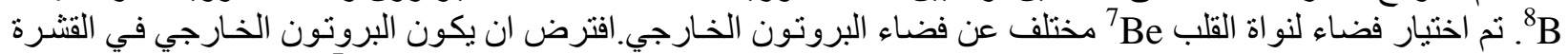

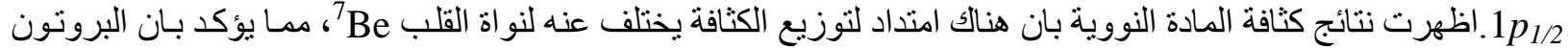

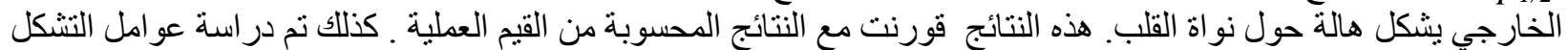

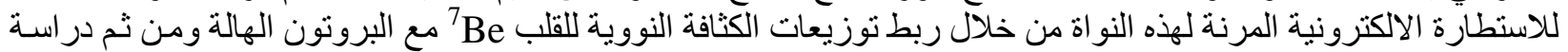

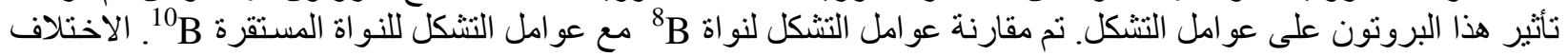

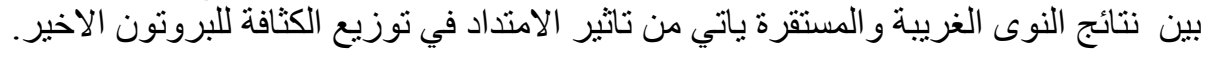

\section{Introduction}

Nuclear halo has been an interesting topic in nuclear physics since the discovery of the halo effect in ${ }^{11} \mathrm{Li}$ [1]. Halo nuclei have extraordinarily large radii and reaction cross sections, so they play an important role in nuclear structure and reactions. A lot of works have been devoted to study the properties of halo nuclei [2-7]. From the theoretical point of view, nuclear halo is a quantum effect, and it arises from extraordinarily extended distribution of weakly bounded valence nucleons [8].

Many attempts were made to look for proton halo in proton drip line nuclei. 
Calculations using different models $[9,10]$ demonstrate that there may be proton halo in the ground state of ${ }^{26-27} \mathrm{P},{ }^{8} \mathrm{~B},{ }^{17} \mathrm{Ne}$ and in the excited states of ${ }^{6} \mathrm{Li}$ and ${ }^{17} \mathrm{~F}$. One of the candidates for a proton- halo nucleus is ${ }^{8} \mathrm{~B}$. The experimental results for its structure are somewhat controversial [11]. Depending on the experimental method and the energy of the incident beam different results are obtained. Measurements of the total interaction and reaction cross sections at energies above $\approx 140 \mathrm{MeV} / \mathrm{u}$ did not give evidence for an extended matter distribution in ${ }^{8} \mathrm{~B}$ [12-14], while later measurements of total reaction cross sections at $20-60 \mathrm{MeV} / \mathrm{u}$ were interpreted as evidence for a long lowdensity tail in the density distributions [15]. The measurements of the momentum distributions of the break-up fragments show narrow peaks which also supports a halo structure $[16,17]$.

Karataglidis et al. [18] studied the elastic electron scattering form factors, longitudinal and transverse, from the $\mathrm{He}$ and $\mathrm{Li}$ isotopes and from ${ }^{8} \mathrm{~B}$. Large space shell model wave functions have been assumed. They emphasized that the effect of the proton halo, in the case of ${ }^{8} \mathrm{~B}$, is very evident with the decrease in the form factor with momentum transfer reflecting the more extensive charge distribution.

The current experimental techniques for recognizing neutron halo and proton halo are mostly based on the measurement of reaction cross sections of the nucleusnucleus collision and of the momentum distributions of nucleus breakup. There are complex processes where the strong and electromagnetic interactions among nucleons play a role. Despite the fact that this type of experiment has achieved most important success for halo phenomena, it is motivating to look for a new probe to refine the study of neutron halo in neutron richnuclei. Electron-nucleus scattering has been confirmed to be tremendous tool for the study of nuclear structure, particularly for the study of electromagnetic properties of nuclei. It has given much consistent information on proton density distributions of stable nuclei. The electron-nucleus scattering is a better technique for the accurate study of the long tail behavior in the nucleon density distribution of the exotic rich nuclei.

There has been no detailed study of elastic electron scattering, in terms of the two frequency shell model, on unstable proton- rich exotic nuclei. Thus, in the present work, form factors of exotic ${ }^{8} \mathrm{~B}$ nucleus are studied with PWBA corresponding to the density distributions obtained by the two-frequency shell model. Effects of the long tail behavior presented in the proton density distribution on the form factors of ${ }^{8} \mathrm{~B}$ are investigated.

\section{Theory}

The form factors for electron scattering between nuclear states $J_{i}$ and $J_{f}$ involving angular momentum transfer $J$ are expressed as [19]

$$
F_{J, t_{z}}(q)=\sqrt{\frac{4 \pi}{2 J_{i}+1}} \frac{1}{N_{t_{z}}}\left\langle J_{f}\left\|\sum_{i=l}^{N_{t_{z}}} \hat{T}_{J, t_{z}}(q, i)\right\| J_{i}\right\rangle,
$$

where the normalization factor $N_{J, t_{z}}$ is defined as

$$
N_{t_{z}}=\left\{\begin{array}{c}
Z \text { for } \mathrm{t}_{\mathrm{z}}=1 / 2 \text { (protons) } \\
N \text { for } \mathrm{t}_{\mathrm{z}}=-1 / 2 \text { (neutrons) }
\end{array}\right\},
$$

and $q$ is the momentum transfer. The longitudinal (Coulomb) multipole operator $\hat{T}_{J, t_{z}}$ is given by [20]

$\hat{T}_{J, t_{z}}(q, i)=\int d^{3} r j_{J}(q r) Y_{J M}(\Omega) \hat{\rho}_{t_{z}}(\vec{r}, i)$, 
where $j_{J}(q r)$ is the spherical Bessel function and $\hat{\rho}_{t_{z}}(\vec{r}, i)$ is the protons/neutrons density operator which is given by

$\hat{\rho}_{t_{z}}(\vec{r}, i)=\delta\left(\vec{r}-\vec{r}_{i}\right)$,

Equation (3) reduced to

$\hat{T}_{J, t_{z}}(q, i)=j_{J}\left(q r_{i}\right) Y_{J M}\left(\Omega_{i}\right)$,

The reduced matrix element in Eq.(1) can be written as a sum over elements of the one-body density matrix (OBDM) elements and the corresponding reduced singleparticle matrix element [21]

$\left\langle\mathrm{J}_{\mathrm{f}}\left\|\sum_{\mathrm{i}=1}^{N_{\mathrm{t}_{2}}} \hat{\mathrm{T}}_{\mathrm{J}, \mathrm{t}_{\mathrm{z}}}\right\| \mathrm{Ji}\right\rangle=\sum_{\mathrm{a}, \mathrm{b}} \operatorname{OBDM}\left(\mathrm{J}_{\mathrm{i}}, \mathrm{J}_{\mathrm{f}}, \mathrm{a}, \mathrm{b}, \mathrm{J}, \mathrm{t}_{\mathrm{z}}\right)\left\langle\mathrm{a}\left\|\hat{\mathrm{T}}_{\mathrm{J}, \mathrm{t}_{2}}\right\| \mathrm{b}\right\rangle$ where $a$ and $b$ label the single particle states $\left(a \equiv n_{a} \ell_{a} j_{a}\right.$ and $\left.b \equiv n_{b} \ell_{b} j_{b}\right)$. The OBDM is defined as [21]

$\operatorname{OBDM}\left(\mathrm{J}_{\mathrm{i}}, \mathrm{J}_{\mathrm{f}}, \mathrm{a}, \mathrm{b}, \mathrm{J}, \mathrm{t}_{\mathrm{z}}\right)=\frac{\left\langle\mathrm{J}_{\mathrm{f}}\left\|\left[\mathrm{a}_{\mathrm{a}, \mathrm{t}_{\mathrm{z}}}^{+} \otimes \tilde{\mathrm{a}}_{\mathrm{b}, \mathrm{t}_{z}}\right]^{(\mathrm{J})}\right\| \mathrm{J}_{\mathrm{i}}\right\rangle}{\sqrt{2 \mathrm{~J}+1}},(7)$

The reduced single-particle matrix elements of the Coulomb operator in equation (6) become:

$$
\left\langle\mathrm{a}\left\|\hat{\mathrm{T}}_{\mathrm{J}}\right\| \mathrm{b}\right\rangle=\int_{0}^{\infty} \mathrm{drr}^{2} \mathrm{j}_{\mathrm{J}}(\mathrm{qr})\left\langle\mathrm{j}_{\mathrm{a}}\left\|\mathrm{Y}_{\mathrm{J}}\right\| \mathrm{j}_{\mathrm{b}}\right\rangle \mathrm{R}_{\mathrm{n}_{\mathrm{a}} \ell_{\mathrm{a}}}(\mathrm{r}) \mathrm{R}_{\mathrm{n}_{\mathrm{b}} \ell_{\mathrm{b}}}(\mathrm{r})
$$

where $R_{n \ell}(r)$ is the harmonic oscillator radial wave function.

The Coulomb form factor given in Eq.(1) becomes:

$F_{J, t_{z}}(q)=\frac{1}{N_{t_{z}}} \sqrt{\frac{4 \pi}{2 J_{i}+1}} \sum_{a, b} \operatorname{OBDM}\left(J_{i}, J_{f}, a, b, J, t_{z}\right)\left\langle j_{a}\left\|Y_{J}\right\| j_{b}\right\rangle \int_{0}^{\infty} d r r^{2} R_{n_{a} \ell_{a}}(r) R_{n_{b} \ell_{b}}(r) j_{J}(q r)$

and can be written as

$F_{J, t_{z}}(q)=\frac{4 \pi}{N_{t_{z}}} \int_{0}^{\infty} d r r^{2} j_{J}(q r) \rho_{J, t_{z}}(r)$

where $\rho_{J, t_{z}}(r)$ is nucleon density

distribution and is given by [21]

$\rho_{J, t_{z}}(r)=\frac{1}{\sqrt{4 \pi}} \frac{1}{\sqrt{2 J_{i}+1}} \sum_{a, b} \operatorname{OBDM}\left(J_{i}, J_{f}, a, b, J, t_{z}\right)\left\langle j_{a}\left\|Y_{J}\right\| j_{b}\right\rangle R_{n_{a} \ell_{a}}(r) R_{n_{b} \ell_{b}}(r)$,

As the model space wave functions have $\operatorname{OBDM}\left(\mathrm{t}_{\mathrm{z}}\right)=(-1)^{\mathrm{T}_{\mathrm{f}}-\mathrm{T}_{z}} \sqrt{2}\left(\begin{array}{ccc}\mathrm{T}_{\mathrm{f}} & 0 & \mathrm{~T}_{\mathrm{i}} \\ -\mathrm{T}_{\mathrm{z}} & 0 & \mathrm{~T}_{\mathrm{z}}\end{array}\right) \operatorname{OBDM}(\mathrm{T}=0) / 2$ good isospin, it is appropriate to evaluate the OBDM elements by means of isospinreduced matrix elements. The relation between the triply reduced OBDM and the proton or neutron OBDM is [21]

$$
+2 \mathrm{t}_{\mathrm{z}}(-1)^{\mathrm{T}_{\mathrm{f}}-\mathrm{T}_{z}} \sqrt{6}\left(\begin{array}{ccc}
\mathrm{T}_{\mathrm{f}} & 1 & \mathrm{~T}_{\mathrm{i}} \\
-\mathrm{T}_{\mathrm{z}} & 0 & \mathrm{~T}_{\mathrm{z}}
\end{array}\right) \operatorname{OBDM}(\mathrm{T}=1) / 2
$$

where the triply reduced $\operatorname{OBDM}(T)$ elements are given by [21] 
$\operatorname{OBDM}\left(J_{i}, J_{f}, \alpha, \beta, J, T\right)=\frac{\left\langle\Gamma_{f}\left\|\left[a_{\alpha}^{+} \otimes \tilde{a}_{\beta}\right]^{J, T)}\right\| \Gamma_{i}\right\rangle}{\sqrt{2 J+1} \sqrt{2 T+1}}$,

Here, Greek symbols are utilized to indicate quantum numbers in coordinate space and isospace

$\left(\alpha \equiv a t_{a}, \beta \equiv b t_{b}, \Gamma_{i}=J_{i} T_{i}\right.$ and $\left.\Gamma_{f}=J_{f} T_{f}\right)$

The OBDM elements contain all the information about transitions of given multi polarities which are embedded in the model space wave functions. Shell model calculations are performed using the code OXBASH [22] with specified model space and effective interaction to generate the OBDM elements.

For the ground state $J=0$ density distribution, we have $J_{i}=J_{f}$ and $\ell_{\mathrm{a}}=\ell_{b}, j_{a}=j_{b}$.

The ground state density distribution takes the form

$\rho_{t_{z}}(r)=\frac{1}{4 \pi} \frac{1}{\sqrt{2 J_{i}+1}} \sum_{a, b} \operatorname{OBDM}\left(J_{i}, J_{i}, a, b, J=0, t_{z}\right) \sqrt{2 j_{a}+1} R_{n_{a} l_{a}}(r) R_{n_{a} l a}(r)$,

The normalization condition of the above ground state density is:

$$
4 \pi \int_{0}^{\infty} d r r^{2} \rho_{t_{z}}(r)=N_{t_{z}},
$$

and the elastic $J=0$ form factor at $q=0$, is equal to one.

As the halo nuclei are oversized and easily broken system consisting of a compact core plus a number of outer nucleons loosely bound and especially extended far from the core, it is suitable to separate the ground state density distribution of Eq. (14) into two parts, one is connected with the core nucleons and the other one with the hole nucleons, so the matter density distribution for the whole halo nucleus becomes:

$$
\rho_{m}(r)=\rho_{p+n}^{\text {core }}(r)+\rho_{p(n)}^{\text {halo }}(r),
$$

The corresponding rms radii are given by[23]

$$
\left\langle r^{2}\right\rangle_{g}^{1 / 2}=\frac{4 \pi}{g} \int_{0}^{\infty} d r r^{4} \rho_{g}(r)
$$

where $\mathrm{g}$ represents the corresponding number of nucleons in each case.

The corresponding elastic scattering $J=0$ form factor $(C 0)$ is written in the following form [20]

$$
F_{0, g}(q)=\frac{4 \pi}{g} \int_{0}^{\infty} d r r^{2} j_{0}(q r) \rho_{g}(r) \text {, }
$$

The average occupation number in each orbit $\left(n_{a, t_{z}}\right)$ is given by

$n_{a, t_{z}}=\sqrt{\frac{2 j_{a}+1}{2 J_{i}+1}} \operatorname{OBDM}\left(J_{i}, J_{i}, a, a, 0, t_{z}\right)$,

The total longitudinal form factor is given by

$$
|F(q)|^{2}=\sum_{J}\left|F_{J}\right|^{2}
$$

\section{Results and Discussion}

One of the candidates for a proton- halo nucleus is ${ }^{8} \mathrm{~B}$, as its last proton has a binding energy of only $137 \mathrm{keV}$. The charge, neutron and matter densities for this nucleus can be calculated in the framework of the two frequency shell model, with two different oscillator size parameters $b_{\text {core }}$ for 
the core orbits and $b_{\text {halo }}$ for the proton halo orbit. Also, electron scattering form factors can be calculated for this nucleus using PWBA.

The proton drip line ${ }^{8} \mathrm{~B}\left(\mathrm{~J}^{\pi} \mathrm{T}=2^{+} 1\right)$ has halo structure, which coupled a ${ }^{7} \mathrm{Be}$ core $\left(\mathrm{J}^{\pi} \mathrm{T}=3 / 2^{-} 1 / 2\right)$ and outer one proton $\left(\mathrm{J}^{\pi} \mathrm{T}=1 / 2^{-} 1 / 2\right)$ forming the ${ }^{8} \mathrm{~B}$. We choose different model spaces for the core and the extra one proton. The configurations $\left(1 s_{1 / 2}\right)^{4}$, $\left(1 p_{3 / 2}\right)^{3}$ are used for ${ }^{7} \mathrm{Be}$. A value of $b_{\text {core }}$ $=1.68 \mathrm{fm}$ is chosen for ${ }^{7} \mathrm{Be}$, which gives the rms matter radius equal to $2.33 \mathrm{fm}$, which is consisting with the measured value $2.33 \pm 0.01 \mathrm{fm}[11]$. The one proton halo is assumed to be in a pure $1 p_{1 / 2}$ orbit. To reproduce the matter radius of ${ }^{8} \mathrm{~B}(2.6 \pm 0.02$ $\mathrm{fm}$ [11]), a value of $b_{\text {halo }}=2.59 \mathrm{fm}$ is chosen for the extra one proton. This value of $b_{\text {halo }}$ gives the matter radius of ${ }^{8} \mathrm{~B}$ equal to 2.618 $\mathrm{fm}$, which is consistent with the measured value.

The matter density distributions $\rho_{\mathrm{m}}$ (r) (in $\mathrm{fm}^{-3}$ ) of ${ }^{8} \mathrm{~B}$ are displayed in Fig.1(a) as a function of $r$ (in $\mathrm{fm}$ ). In this figure, the solid line is the calculated matter density obtained when the outer one proton is in a pure $1 p_{1 / 2}$ orbit. The filled circles are the fitted matter densities taken from ref. [11] for the Gaussian-Gaussian (G-G) parameterization. It is evident from this figure that the calculated matter density distribution is in an excellent accordance with the fitted data. A long tail behavior, which is a distinctive feature of the halo nuclei, is evidently revealed in these calculated distributions. Fig.1(b) shows the core (proton+ neutron) (dashed line) and one halo proton (dashdotted line) contributions to the matter density of ${ }^{8}$ B. Fig.2(a) shows the calculated proton and neutron density displayed as dashed and dash-dotted lines, respectively.
The long tail behavior is noticeably seen in the distribution of the proton. This behavior is related to the existence of the outer proton of ${ }^{8} \mathrm{~B}$ in the halo orbit. The difference between the proton and neutron rms radii is $\mathrm{R}_{\mathrm{p}}-\mathrm{R}_{\mathrm{n}}=2.805-2.274=0.531 \mathrm{fm}$, which provides an additional evidence for the halo structure of these nuclei. The calculated neutron density distributions shown in this figure demonstrate a steep slope behavior because there are no neutrons found in the halo orbit.

To seek out if the long tail behavior of the proton density distributions of ${ }^{8} \mathrm{~B}$ proton rich nucleus demonstrates noticeable effects in the process of elastic electron scattering, elastic form factors of unstable ${ }^{8} \mathrm{~B}$ nucleus and its stable isotope ${ }^{10} \mathrm{~B}$ nucleus (same number of protons) are calculated by means of PWBA. To compare the results of the form factor of ${ }^{8} \mathrm{~B}$ with that of the stable isotope, we first present the calculated $(C 0+C 2)$ form factor of ${ }^{10} \mathrm{~B}$ (solid line). Same model space is used as for the ${ }^{7} \mathrm{Be}$ core nucleus. We choose a size parameter for the harmonic oscillator radial wave functions $b=1.724 \mathrm{fm}$ for ${ }^{10} \mathrm{~B}$ to reproduce the matter radius, $2.4829 \pm 0.0019 \mathrm{fm}$ from a very precise muonic X-ray experiment [24]. These results are displayed in Fig.2(b), using effective charges equal to $1.35 \mathrm{e}$ and $.35 \mathrm{e}$, for the protons and neutrons, respectively, for $C 2$ to account for the core polarization effects [21]. Also, center of mass and finite nucleon size corrections are introduced. The experimental data of ref. [25] are very well reproduced by our calculations. The decompositions of the form factor into $C 0$ and $C 2$ are shown in this figure by the dotted and dashed lines, respectively. 

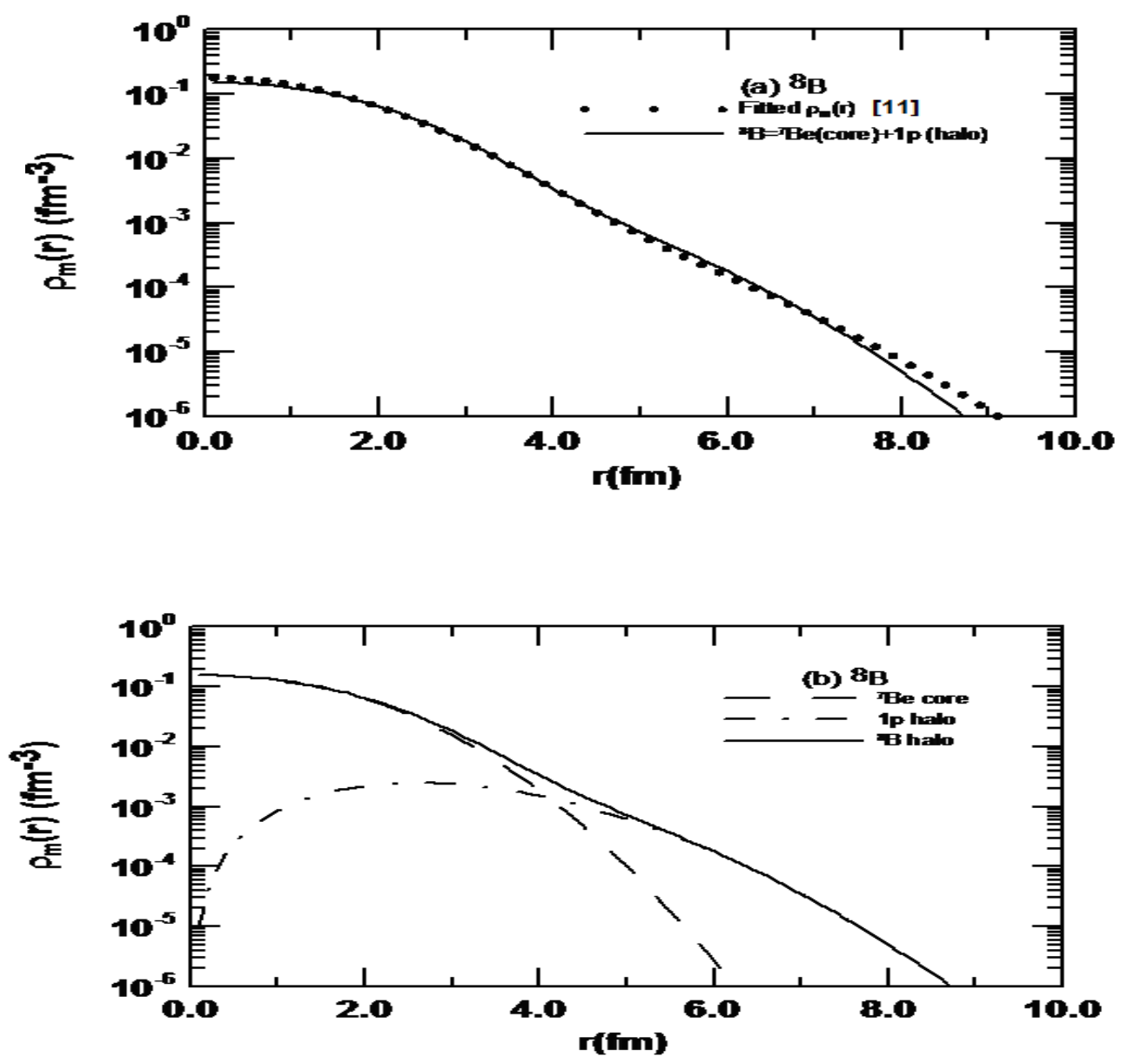

Fig.1: Matter density distributions of ${ }^{8}$ B nucleus.

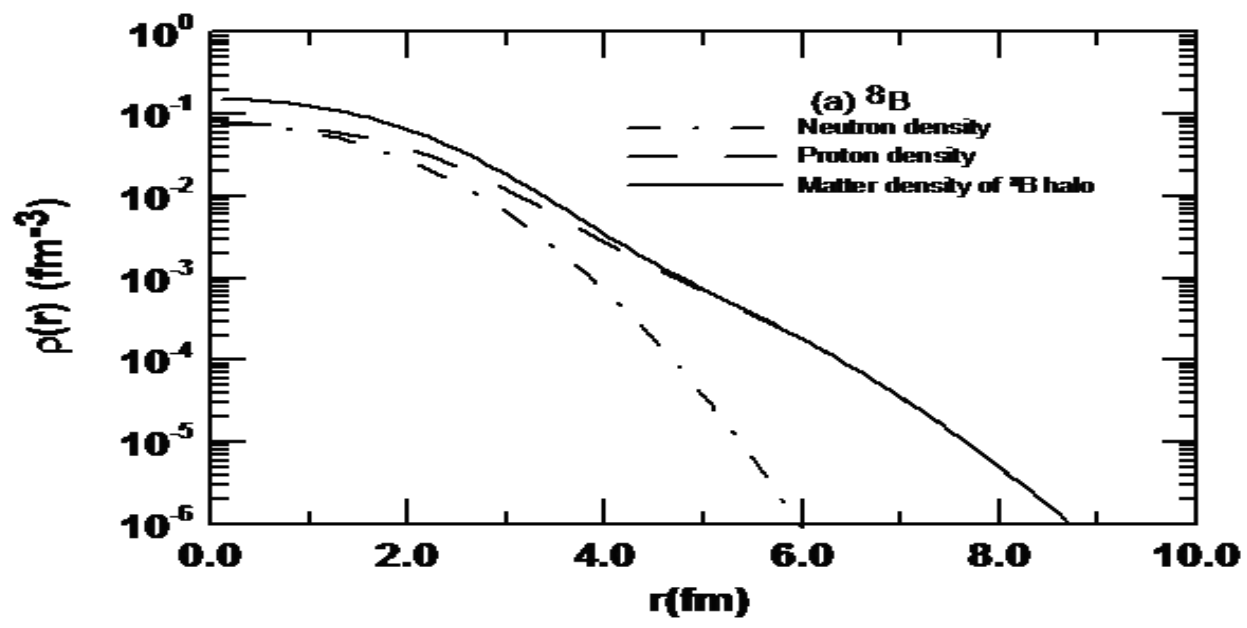

Fig.2: (a) The contributions of proton and neutron density to matter density of ${ }^{8} B$ halo nucleus. 


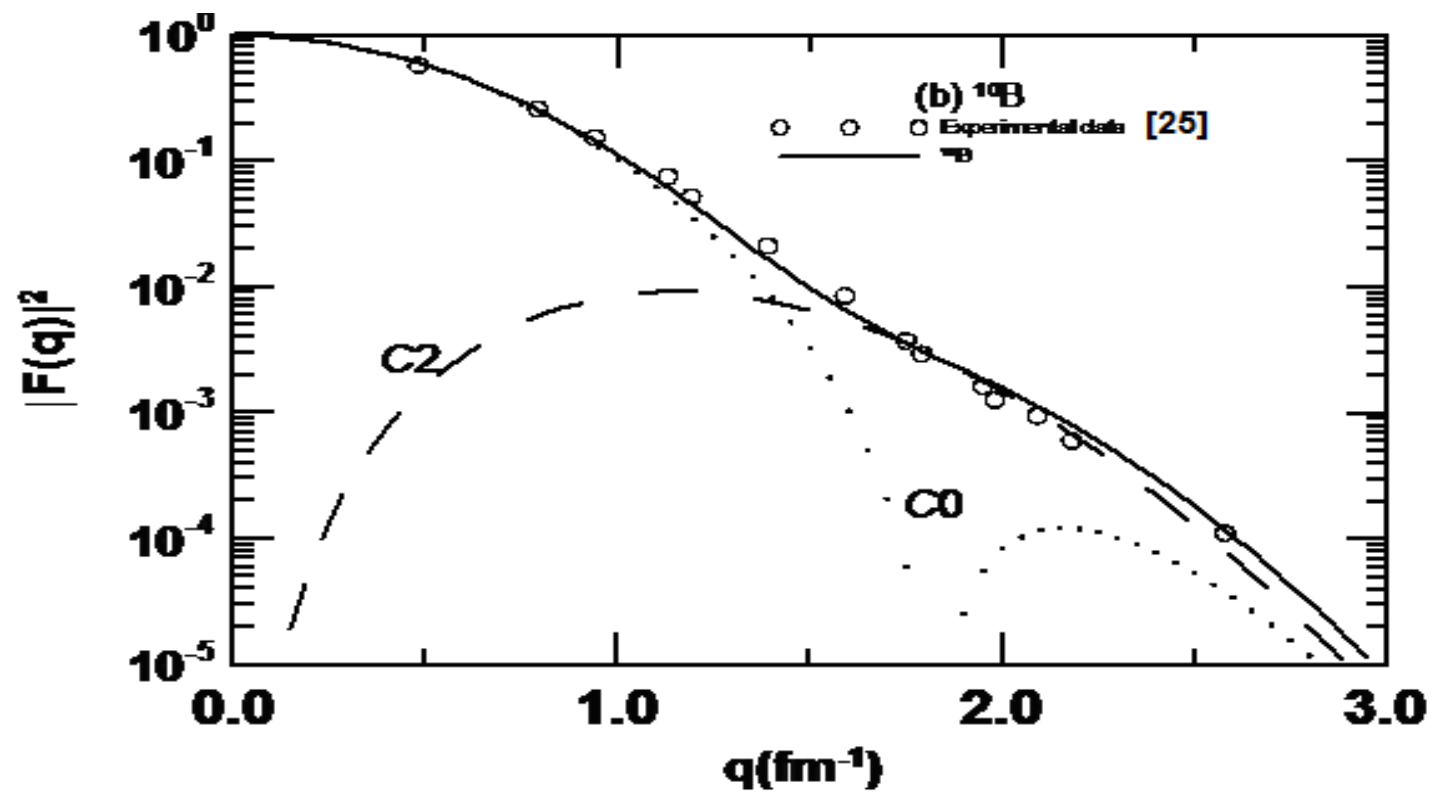

Fig.2: (b) Charge form factor of ${ }^{10} \mathrm{~B}$ (solid line) and its decompositions into CO (dotted line) and C2 (dashed line) contributions in comparison with the experimental data (open circles) of ref. [25].

The charge density corresponding to $C 0$ form factor is displayed in Fig.3(a) as dashed line in comparison with that of ${ }^{8} \mathrm{~B}$ (solid line). It is seen from this figure that the charge density distributions of ${ }^{8,10} \mathrm{~B}$ are different although the two nuclei have the same proton number. The proton form factors for ${ }^{8} \mathrm{~B}$ halo nucleus and ${ }^{10} \mathrm{~B}$ stable nucleus are shown in Fig. 3(b), as solid line and dashed line, respectively. For the sake of comparison, neither core polarization effects nor center of mass and finite nucleon size corrections are considered for those form factors. Comparing the result of ${ }^{8} \mathrm{~B}$ with that of ${ }^{10} \mathrm{~B}$, shows that the form factor for ${ }^{8} \mathrm{~B}$ in the range of $q>2 \mathrm{fm}^{-1}$ is decreased from that of ${ }^{10} \mathrm{~B}$ which shows that the form factor in this range of $q$ is sensitive to the charge density distributions near the center of the nucleus [26], while for $\mathrm{q}<2 \mathrm{fm}^{-1}$, the form factor is not sensitive to the modification of the charge distribution.
These behaviors are clearer for $C 0$ form factors as shown in Fig. 4, since the ground state charge density is connected with $C 0$ form factor. These form factors are connected with the charge density distributions of ${ }^{8,10} \mathrm{~B}$, given in Fig. 3(a). The position of the minimum is sensitive to the charge density distribution around the center of the nucleus, while the form factor for low $q$ values is insensitive to the density distribution around the center of the nucleus. The significant difference between the form factor of the exotic proton-drip line ${ }^{8} \mathrm{~B}$ and that of the stable ${ }^{10} \mathrm{~B}$ is attributed to the influence of the charge density distribution of the last proton in ${ }^{8} \mathrm{~B}$. The $C 0$ form factors in the range of momentum transfer $1.5 \leq q \leq 3 \mathrm{fm}^{-1}$ are sensitive to the change of the tail part of the charge density, while those at high momentum transfers to the change of the inner part of the charge distribution. These conclusions are 
consisting with those of ref. [27] for ${ }^{12} \mathrm{C}$ and of ref. [26] for $\mathrm{O}$ and $\mathrm{S}$ isotopes. For $\mathrm{C2}$ contribution, the form factors of ${ }^{8} \mathrm{~B}$ and ${ }^{10} \mathrm{~B}$ are almost identical, because the ground state charge density has nothing to do with $C 2$ form factor.
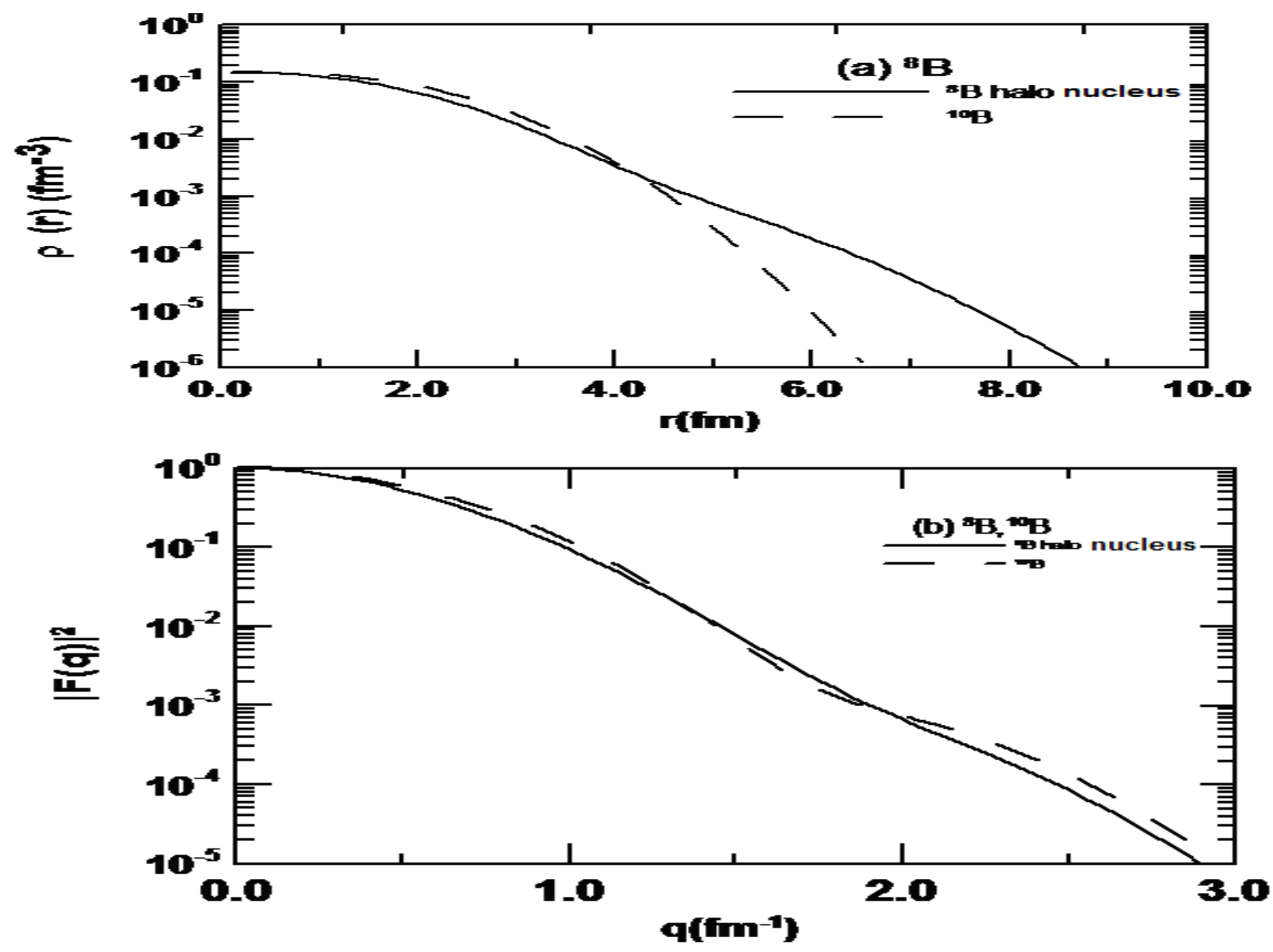

Fig.3: (a) Charge density distribution of ${ }^{8} B$ halo nucleus (solid line) in comparison with that of its stable isotope ${ }^{10} B($ dashed line).

(b) Charge form factor of ${ }^{8} B$ (halo) nucleus (solid line) in comparison with that of its stable ${ }^{10} B$ nucleus (dashed line).

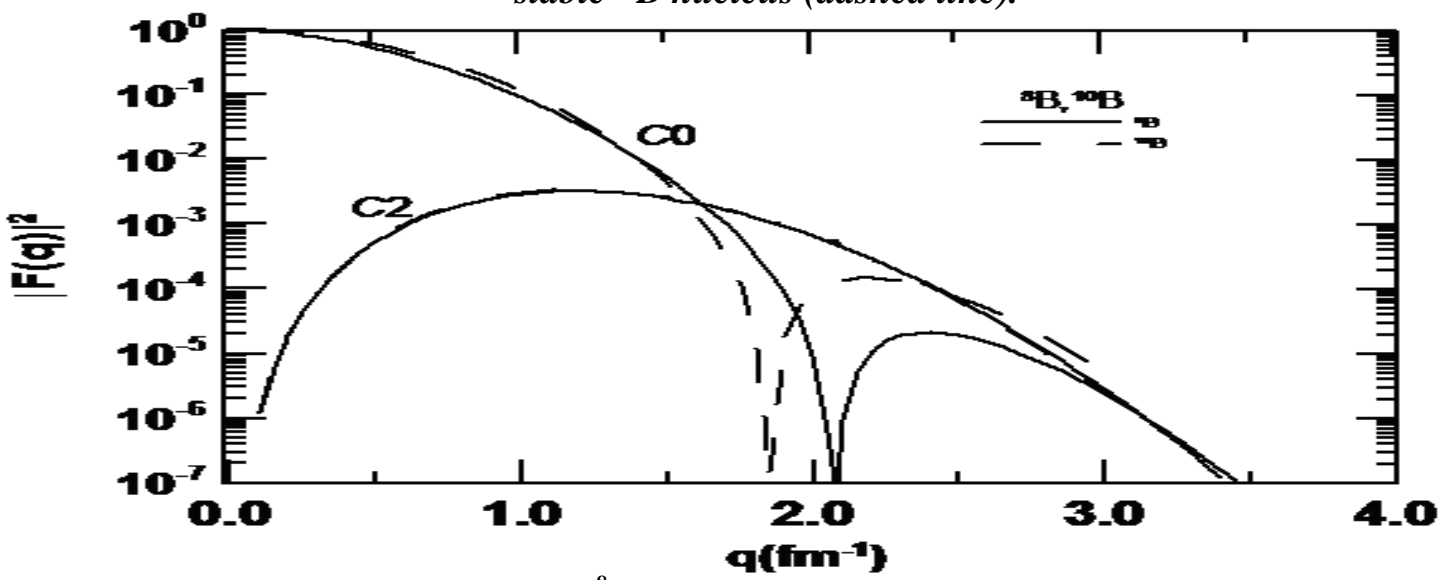

Fig.4: $\mathrm{CO}$ and $\mathrm{C} 2$ form factors of ${ }^{8} \mathrm{~B}$ (halo) nucleus (solid lines) in comparison with those of ${ }^{10} \mathrm{~B}$ nucleus (dashed lines). 


\section{Conclusions}

The ground state proton, neutron and matter density distributions of the unstable proton -rich ${ }^{8} \mathrm{~B}$ nucleus are investigated using the two-frequency shell model approach. Elastic electron scattering from this exotic nucleus are also investigated. The long tail behavior, considered as a distinctive feature of halo nuclei, is evidently revealed in the calculated proton and matter density distributions of this exotic nucleus. Besides, the noticeable difference that is found between the calculated overall proton and neutron rms radii also indicates a definite degree of halo structure. It is found that the structure of the halo proton for this nucleus has $1 p_{1 / 2}$ configuration. Also, it is found that the difference between the form factors of ${ }^{8} \mathrm{~B}$ nucleus and stable ${ }^{10} \mathrm{~B}$ nucleus is mainly caused by the long tail behavior present in the proton density distributions of ${ }^{8} \mathrm{~B}$. The $\mathrm{C} 0$ form factors in the range of momentum transfer $1.5 \leq q \leq 3 \mathrm{fm}^{-1}$ are sensitive to the change of the tail part of the charge density, while those at high momentum transfers to the change of the inner part of the charge distribution.

\section{References}

[1] I. Tanihata, H. Hamagaki, O. Hashimoto, Y. Shida, N. Yoshikawa, K. Sugimoto, O.

Yamakawa and T. Kobayashi, Phys. Rev. Lett. 55 (1985) 2676.

[2] P. G. Hansen and J. A. Tostevin, Ann. Rev. Nucl. Part. Sci. 53 (2003) 219.

[3] A. S. Jensen, K. Riisager, D.V. Fedorov and E. Garrido, Rev. Mod. Phys., 76 (2004) 215.

[4] W. Mittig, J. M. Chouvel, Z.W. Long, L. Bianchi, A. Cunsolo, B. Fernandez, A. Foti, J. Gastebois, A. Gillibert, C. Gregoire, Y. Schutz and C. Stehpan, Phys. Rev. Lett. 59 (1987) 1889.
[5] Y. T. Oganessian, V. I. Zagrebaev and J. S. Vaagen, Phys. Rev. Lett. 82 (1999) 4996. [6] W. J. Guo, H. Q. Hian, J. Y. Liu, W. Zuo, Z. Z. Ren and X. G. Lee, Commum. Theor.Phys., 40 (2003) 577.

[7] B. P. Gu and Z. Z. Ren, Commum. Theor. Phys., 46 (2006) 711.

[8] CHU Yan-Yun, REN Zhong-Zhou and WANG Zai-Jun, Commun.Theor.Phys.54 (2010) 345.

[9] Z. Ren, B. Chen, Z. Ma and G. Xu, Phys. Rev.C53 (1996) R572.

[10] B. A. Brown and P. G. Hansen, Phys. Lett. B 381 (1996) 391.

[11] S.Ilieva, Ph.D. Thesis, der Johannes Gutenberg-University, Mainz, (2008).

[12] B. Blank C. Marchand, M. S. Pravikoff, T. Baumann, F. Boue, H. Geissel, M.

Hellstrom, N. Iwasa, W.Schwab, K. Summerer and M.Gai, Nucl. Phys., A624 (1997) 242.

[13] I. Pecina, R. Anne, D. Bazin, C. Borcea,V. Borrel, F. Carstoiu, J. M. Corre, Z.Dlouhy, A. Fomitchev, D. GuillemaudMueller, H. Keller, A. Kordyasz, M.Lewitowicz, S. Lukyanov, A. C. Mueller, Yu. Penionzhkevich, P. Roussel-Chomaz,M. G. Saint-Laurent, N.Skobelev, O. Sorlin and O. Tarasov, Phys. Rev. C52 (1995)191.

[14] M. M. Obuti, T. Kobayashi, D. Hirrata, Y. Ogawa, A. Ozawa, K. Sugimoto, I.Tanihata, D. Olson, W. Christie and H. Wieman Nucl. Phys.A609 74.

[15] R. E. Warner, J. H. Kelley, P.Zecher, F. D. Becchetti, J. A. Brown, c. 1. carpenter, A.Galonsky, J. Kruse, A. Muthukrishnan, A.Nadasen, R. M. Ronningen, P. Schwandt, B. M. Sherrill, J. Wang and J. S. Winfild, Phys. Rev. C 52 (1995)R1166; M. Fukuda, M. Mihara, T. Fukao, S. Fukuda, M. Ishihara, S. Ito, T. Kobayashi, K. Matsuta, T.Minamisono, S. Momota, T. Nakamura, Y. Nojiri, Y. Ogawa, T. Oohtsubo, T. 
Onishi, A. Ozawa, T. Suzuki, M. Tanigaki, I. Tanihata and K. Yoshida Nucl. Phys., A656 (1999) 209.

[16] M. H. Smedberg, T. Baumann, T. Aumann, L. Axelsson, U. Bergmann, M. J. G.Borge, D. Cortina-Gil, L. Fraile, H. Geissel, L. Grigorenko, M. Hellstraom, M. Ivanov, N. Iwasa, R. Janik, B. Jonson, H. Lenske, K. Markenroth, G. Maunzenberg, T. Nilsson, A. Richter, K. Riisager, C. Scheidenberger, G. Schrieder, W. Schwab, H.Simon, B. Sitar, P. Strmen, K. Saummerer, M. Winkler and M. V. Zhukov Phys. Lett.B452 (1999) 1.

[17] C. Borcea, F. Carstoiu, F. Negoita, M. Lewitowicz, M. G. Saint-Laurent, R. Anne, D.Bazin, V. Borrel, J. M. Corre, Z.Dlouhy, A. Fomitchev, D. Guillemaud-Mueller, H. Keller, A. Kordyasz, S. Lukyanov, A.C.Muller, Yu. Penionzhkevich, P. Roussel- Chomaz, N. Skobelev, O. Sorlin Nucl. Phys. A616 (1997) 231.

[18] S. Karataglidis and K. Amos, Phys. Lett. B 650 (2007) 148.

[19] T. deforest, Jr. and J. D. Walecka, Adv. Phys., 15 (1966)1.
[20] H. G. Benson and B. H. Flowers; Nucl. Phys., A126 (1969) 305.

[21] B. A. Brown, R. Radhi and B. H. Wildenthal, Physics Reports 101 (1983)313. [22] B. A. Brown, A. Etchegoyen, N. S. Godwin, W. D. M. Rae, W. A. Richter, W. E.Ormand, E. K. Warburton, J. S. Winfield, L. Zhao and C. H. Zimmerman, Oxbash for windows, MSU- NSCL report number 1289 2005 version.

[23] I.S.Towner,"A shell model description of light nuclei", Clarendon press, Oxford, (1971).

[24] W. Ruskstuhl, B. Aas, W. Beer, I. Beltrami, K. Bos, P. F. A. Goudsmit, H. J. Leisi, G. Strassner, A. Vacchi and F. W. N. De Boer Nucl. Phys., A430 (1985) 685.

[25] A. Cichocki, J. Dubach, R. S. Hicks, G. A. Peterson, C. W. de Jager, H. de Vries,N. Kalantar-Nayestanaki and T.Sato, Phys. Rev. C 51 (1995) 2406.

[26] Zaijum Wang and Zhongzhou Ren, Phys. Rev. C 70 (2004) 034303.

[27] I. Sick and J. S. McCarthy, Nucl. Phys., A150 (1970) 631. 